\title{
Associação entre comportamento alimentar, consumo de cigarro, drogas e episódios depressivos em adolescentes
}

\author{
Association between eating behavior and smoking, \\ use of illicit drugs and depressive \\ episodes in adolescents
}

Juliany Piazzon GOMES'

Elto LEGNANI ${ }^{2}$

Rosimeide Francisco dos Santos LEGNANI ${ }^{3}$

Nicolly Patrícia GREGÓRIO4

Raphael Klein de SOUZA²

RE S U M O

\section{Objetivo}

Identificar a prevalência de sintomas de transtornos alimentares em estudantes do sexo feminino matriculadas no ensino fundamental da rede estadual do município de Toledo (PR), bem como relacionar esses sintomas com o hábito de fumar, o uso de drogas e episódios depressivos.

\section{Métodos}

Foram utilizados os instrumentos Teste de Investigação Bulímica de Edimburgo, com escala de sintoma e gravidade, e o Eating Attitudes Test-26, acrescido de perguntas sobre tabagismo, uso de drogas e episódios depressivos. A amostra constituiu-se de cerca de 300 estudantes do sexo feminino, na faixa etária entre 10 e 15 anos.

\section{Resultados}

Os resultados demonstraram que as estudantes apresentaram prevalências de sintomas de transtornos alimentares na ordem de 4,2\% para a escala Teste de Investigação Bulímica de Edimburgo e 3,6\% para o Eating Attitudes Test-26. A prevalência de consumo de cigarro foi de 4,2\%, do uso de drogas, 4,9\% e de episódios depressivos, 10,7\%. Observaram-se associações significativas entre episódios depressivos e os seguintes

\footnotetext{
${ }^{1}$ Universidade Tecnológica Federal do Paraná. Campus Londrina, Av. dos Pioneiros, 3131, 86036-370, Londrina, PR, Brasil Correspondência para/Correspondence to: J. PIAZZON-GOMES. E-mail: <jugomes@utfpr.edu.br>.

2 Universidade Tecnológica Federal do Paraná. Toledo, PR, Brasil.

3 Universidade Estadual de Londrina, Centro de Educação Física e Esporte. Londrina, PR, Brasil.

${ }^{4}$ Universidade Paranaense, Curso de Nutrição. Paraná, PR, Brasil.
} 
756 | J. P. GOMES et al.

parâmetros: Teste de Investigação Bulímica de Edimburgo - escala de sintomas $(p=0,04)$ e Teste de Investigação Bulímica de Edimburgo - escala de gravidade $(p=0,02)$. Os hábitos de fumar $(p=0,01)$ e de usar drogas $(p=0,04)$ também apresentaram associação com Teste de Investigação Bulímica de Edimburgo - escala de sintomas.

\section{Conclusão}

Verificou-se nas estudantes deste estudo elevada probabilidade de desenvolver comportamentos de risco, tais como transtornos alimentares e episódios depressivos.

Termos de indexação: Adolescente. Anorexia nervosa. Bulimia nervosa. Transtornos alimentares.

\section{A B S TR A C T}

\section{Objective}

The goal of this study was to identify the prevalence of symptoms of eating disorders in female students enrolled in state elementary schools and investigate a possible association between these eating disorders and smoking, illicit drug use and depressive episodes.

\section{Methods}

The Bulimic Investigatory Test of Edinburgh with symptom and severity scales and the Eating Attitudes Test - 26 with additional questions on smoking, illicit drug use and depressive episodes were used. The sample consisted of roughly 300 female students aged 10 to 15 years.

\section{Results}

The results showed that $4.2 \%$ and $3.6 \%$ of the students presented symptoms of eating disorders according to the Bulimic Investigatory Test of Edinburgh and Eating Attitudes Test - 26, respectively. The rates of smoking, illicit drug use and depressive episodes were 4.2\%, 4.9\% and 10.7\%, respectively. Depressive episodes were significantly associated with the following parameters: Bulimic Investigatory Test of Edinburgh - symptom scale $(p=0.04)$ and Bulimic Investigatory Test of Edinburgh - severity scale $(p=0.02)$. Smoking $(p=0.01)$ and illicit drug use $(p=0.04)$ were also associated with the Bulimic Investigatory Test of Edinburgh - symptom scale.

\section{Conclusion}

This study found that these students were at high risk of developing risky behaviors, such as eating disorders and depressive episodes.

Indexing terms: Adolescent. Anorexia nervosa. Bulimia nervosa. Eating disorders.

\section{N T R O D U ÇÃ O}

Os adolescentes têm como característica comportamental a contestação, que os torna vulneráveis, volúveis, seguidores de líderes, grupos e modas, desenvolvendo preocupações ligadas ao corpo e à aparência'. Meninas que apresentam ou estão em risco de apresentar desordens alimentares exibem problemas específicos, como insatisfação com a imagem corporal e atitudes inadequadas em relação à comida: restrição a alimentos ou seu consumo excessivo em episódios delimitados (binge eating) e comportamentos compensatórios por comer em excesso².

O medo obsessivo da obesidade faz com que cada vez mais mulheres controlem o peso corporal com o uso de dietas milagrosas, exercícios exagerados, laxantes, diuréticos e drogas anorexígenas. O gênero feminino, geralmente, é o mais vulnerável às pressões sociais, econômicas e culturais associadas aos padrões estéticos e, por isso, é mais suscetível aos transtornos alimentares, representando 95\% dos casos. As formas mais descritas na literatura são: anorexia nervosa, bulimia nervosa e transtorno de compulsão alimentar periódica ${ }^{3}$.

O Manual Diagnóstico e Estatístico de Transtornos Mentais (DSM-IV-TR) $307.51^{4}$ e a Classificação Internacional de Doenças (CID-10) F50.25 (Quadro 1) classificam a bulimia nervosa e a anorexia nervosa separadamente, apesar de os transtornos estarem relacionados intimamente e 
apresentarem como psicopatologia a ideia prevalente relacionada a uma preocupação excessiva com o peso e a forma corporal, que lança o sujeito a procurar dietas restritivas ou procurar métodos inapropriados para alcançarem o "corpo perfeito". Esses indivíduos tendem a julgar a si mesmo com base em sua aparência física, e normalmente a percepção da própria aparência física não Ihes agrada. Concretamente, o DSM-IV4 assinala a prevalência da anorexia nervosa na população adolescente e juvenil feminina entre 0,5 e $1 \%$, e a da bulimia nervosa entre $1 \%$ e $3 \%{ }^{4}$.

A anorexia nervosa e a bulimia nervosa, sendo transtornos de origem multifatorial, necessitam de avaliações e abordagens que contemplem os vários aspectos envolvidos em sua gênese e manutenção. Assim, a aplicação de instrumentos que avaliem as comorbidades (especialmente sintomas depressivos e ansiosos), a imagem corporal, a qualidade de vida e adequação social, além daqueles utilizados para o rastreamento ou diagnóstico do transtorno em si, será de grande utilidade para uma melhor compreensão do quadro clínico e para a elaboração de estratégias de tratamento mais adequadas, que poderão melhorar os indicadores de sucesso terapêutico ${ }^{6}$.
O Eating Attitudes Test (EAT) é utilizado para o rastreamento de indivíduos suscetíveis ao desenvolvimento de anorexia. O teste indica a presença de padrões alimentares anormais, mas não revela a possível psicopatologia subjacente ${ }^{7}$. O Bulimic Investigatory Test Edinburgh (BITE) foi desenvolvido para o rastreamento e a avaliação da gravidade da bulimia e apresentou propriedades psicométricas adequadas no estudo original ${ }^{7}$.

Esta investigação tem como objetivo estimar a prevalência de comportamentos sugestivos de anorexia e bulimia nervosa segundo o EAT-26 e o BITE e relacionar esses transtornos alimentares com o hábito de fumar, usar drogas e episódios depressivos nas estudantes do ensino fundamental do município de Toledo (PR).

\section{M É T O D O S}

Por meio do método de seleção amostral por conglomerados, selecionou-se uma amostra representativa de cerca de 300 adolescentes do sexo feminino, com idades de 10 a 15 anos, matriculadas no ensino fundamental e frequentes em escolas da rede estadual de ensino da cidade de Toledo (PR).

Quadro 1. Comportamento alimentar e depressão. Toledo (PR), 2007.

F50.2 - Anorexia nervosa

(a) Há uma preocupação persistente com o comer e um desejo irresistível de comida; o paciente sucumbe a episódios de hiperfagia, nos quais grandes quantidades de alimento são consumidas em curtos períodos de tempo.

(b) O paciente tenta neutralizar os efeitos "de engordar" dos alimentos através de um ou mais do que se segue: vômitos autoinduzidos; abuso de purgantes; períodos alternados de inanição; uso de anorexígenos, preparados tireoidianos ou diuréticos. Quando a bulimia ocorre em pacientes diabéticos, eles podem escolher negligenciar seu tratamento insulínico.

(c) A psicopatologia consiste de um pavor mórbido de engordar e o paciente coloca para si mesmo um limiar de peso nitidamente definido, bem abaixo de seu peso pré-mórbido, que constitui o peso ótimo ou saudável na opinião do médico. Há frequentemente, mas não sempre, uma história de um episódio prévio de anorexia nervosa, o intervalo entre os dois transtornos variando de poucos meses a vários anos. Esse episódio prévio pode ter sido completamente expressado ou pode ter assumido uma forma 'disfarçada' menor, com uma perda de peso moderada e/ou uma fase transitória de amenorreia.

307.51 - Bulimia nervosa

(a) Crises bulímicas recorrentes.

(b) Comportamento compensatório inadequado e recorrente, com o fim de prevenir o aumento de peso, como indução de vômitos e outros.

(c) A crise bulímica e os comportamentos compensatórios inadequados ocorrem, em média, pelo menos duas vezes por semana, por 3 meses.

(d) A auto-imagem é indevidamente influenciada pela forma e pelo peso do corpo.

(e) O distúrbio não ocorre exclusivamente durante episódios de anorexia nervosa. 
A coleta de dados ocorreu durante os meses de março e maio de 2007. Um dia antes da aplicação do questionário, foram distribuídos os Termos de Consentimento Livre e Esclarecido (TCLE), para que os pais das alunas autorizassem a coleta de dados. No dia seguinte, os pesquisadores apresentaram-se ao professor presente na sala de aula sorteada. Após breve explicação sobre o trabalho, a participante respondeu ao questionário individualmente, não necessitando de identificação pessoal. O tempo médio de preenchimento foi de 30 minutos.

Os questionários para investigar comportamentos bulímicos BITE e de anorexia nervosa (EAT-26) baseiam-se em perguntas pertencentes às escalas validadas mais utilizadas nos estudos sobre transtornos alimentares, em suas respectivas versões em língua portuguesa, sendo elas:

a) Teste de Investigação Bulímica de Edimburgo(BITE) ${ }^{8}$ - tradução de Cordás \& Hochgraf ${ }^{9}$, que permite identificar comedores compulsivos e obter dados sobre aspectos cognitivos e comportamentais da bulimia nervosa.

b) Teste de Atitudes Alimentares resumido $\left(\right.$ EAT-26) ${ }^{10}$ - tradução de Nunes et al. ${ }^{11}$, que avalia os riscos de se desenvolverem comportamento e atitudes típicos de pacientes com anorexia nervosa.

Além de tais escalas, foram aplicadas, anexas ao questionário, perguntas sobre tabagismo, uso de drogas (álcool e outras) e episódios depressivos. O objetivo da aplicação de tais perguntas foi conseguir uma melhor caracterização da amostra por meio dos dados adicionais a fim de relacioná-los com a prevalência de comportamento alimentar sugestivo de anorexia e bulimia nervosa na amostra.

O resultado do BITE foi obtido pela escala de sintomas (BITE - escala de sintomas) e pela escala de gravidade (BITE - escala de gravidade), que mede a gravidade do comportamento compulsivo pela frequência. A classificação das alunas, segundo o BITE, foi realizada de acordo com os seguintes critérios:
Escala de sintomas: 1) Escore maior ou igual a 20: presença de comportamento alimentar compulsivo com grande possibilidade de preencher critérios diagnósticos para bulimia pelo DSM-III-R (4), 2) Escore entre 10 e 19: sugestivo de padrão alimentar não usual. Em geral, não estão presentes todos os critérios para bulimia. Deve ser seguido de entrevista clínica. Escores de 15 a 19 devem ser obrigatoriamente seguidos de entrevista. Podem representar um grupo subclínico de indivíduos com alimentação compulsiva, bulímicos em estágio inicial ou bulímicos em recuperação, 3) Escore abaixo de 10: normalidade.

Escala de gravidade: 1) Escore maior ou igual a 10: indica alto grau de gravidade, 2) Escore maior que 5: indica estado clínico comprometido.

Escores clinicamente significativos devem ser seguidos de entrevista diagnóstica.

A classificação das alunas segundo o EAT-26 foi: 1) Escore maior ou igual a 20: alto risco, 2) Escore de 10 a 19: baixo risco, 3) Escore de 0 a 9: fora de risco. Foi atribuído valor positivo $(E A T+)$ para EAT $>20$ e valor negativo (EAT -) para EAT $<20$.

Os dados foram digitados no programa epidata $2.0^{12}$, realizando-se uma conferência manual de $10 \%$ dos questionários digitados para a verificação de possíveis erros de digitação. Em seguida, foram transportados para o pacote estatístico SPSS versão 15.0 for Windows ${ }^{12}$. As variáveis qualitativas foram analisadas pelo teste do qui-quadrado $\left(\chi^{2}\right)$ e as quantitativas por meio da estatística descritiva (média, desvio-padrão e percentil). Estabeleceu-se um nível de confiança de $95 \%$. Nos casos em que o resultado apresentado não atinge $100 \%$, a diferença é explicada pelas respostas em branco. O projeto de pesquisa recebeu aprovação do Comitê de Ética em Pesquisa da Universidade Paranaense por meio do Parecer No 1013/2007.

RESULTADOS

Com base nos critérios sugeridos pela Organização Mundial da Saúde ${ }^{13}$, as adolescentes 
foram classificadas de acordo com seu estado nutricional: baixo peso $(<\mathrm{p} 5)$, eutróficas ou peso normal (p5 $\geq$ e $<$ p85), sobrepeso (p85 $\geq-\leq p 95)$ ou obesidade (>p95). Cerca de $5,4 \%$ (14) das adolescentes foram classificadas com baixo peso, $82,3 \%$ (235), como eutróficas, 10,8\% ( $n=31)$, com sobrepeso e $5,6 \%(n=12)$, com obesidade. As maiores prevalências de baixo peso $(7,7 \%)$ e obesidade $(7,7 \%)$ foram observadas na idade de 15 anos; com relação ao sobrepeso (14,4\%), as maiores prevalências foram observadas na idade de 14 anos.

Com relação às questões relacionadas ao comportamento alimentar compulsivo do questionário BITE - escala de sintomas, 36,2\% ( $n=109)$ das adolescentes investigadas relataram comportamento alimentar não usual, e 4,2\% comportamento alimentar compulsivo. No entanto, quando avaliadas pelas questões relacionadas à escala de gravidade, BITE - escala de gravidade, 34,2\% $(n=105)$ das adolescentes relataram compor- tamento alimentar comprometido, e 4,2\% ( $n=11)$, comportamento alimentar de alta gravidade.

Os resultados relacionados à prevalência de comportamentos sugestivos de anorexia (EAT) demonstraram que $34,2 \%(n=103)$ das adolescentes foram classificadas como baixo risco, e $3,6 \%(n=11)$ apresentaram alto risco para sintomas de anorexia. A prevalência do consumo de fumo por parte das adolescentes foi de $4,2 \%$ $(n=12)$ e de drogas, de $4,9 \%(n=15)$; já para os episódios depressivos, a prevalência de ocorrência desse sintoma foi de 10,7\% ( $n=32)$ entre as adolescentes.

Para verificar as possíveis associações entre o consumo de fumo, drogas e episódios depressivos com comportamentos sugestivos de anorexia (EAT) e bulimia (BITE - escala de sintomas e BITE - escala de gravidade), optou-se por dicotomizar os dados das variáveis (Tabela 1).

Não foi observada associação significativa do EAT com nenhuma das variáveis analisadas.

Tabela 1. Associação entre comportamento alimentar inadequado (bulimia e anorexia) e consumo de cigarro, drogas e episódios depressivos. Toledo (PR), 2007.

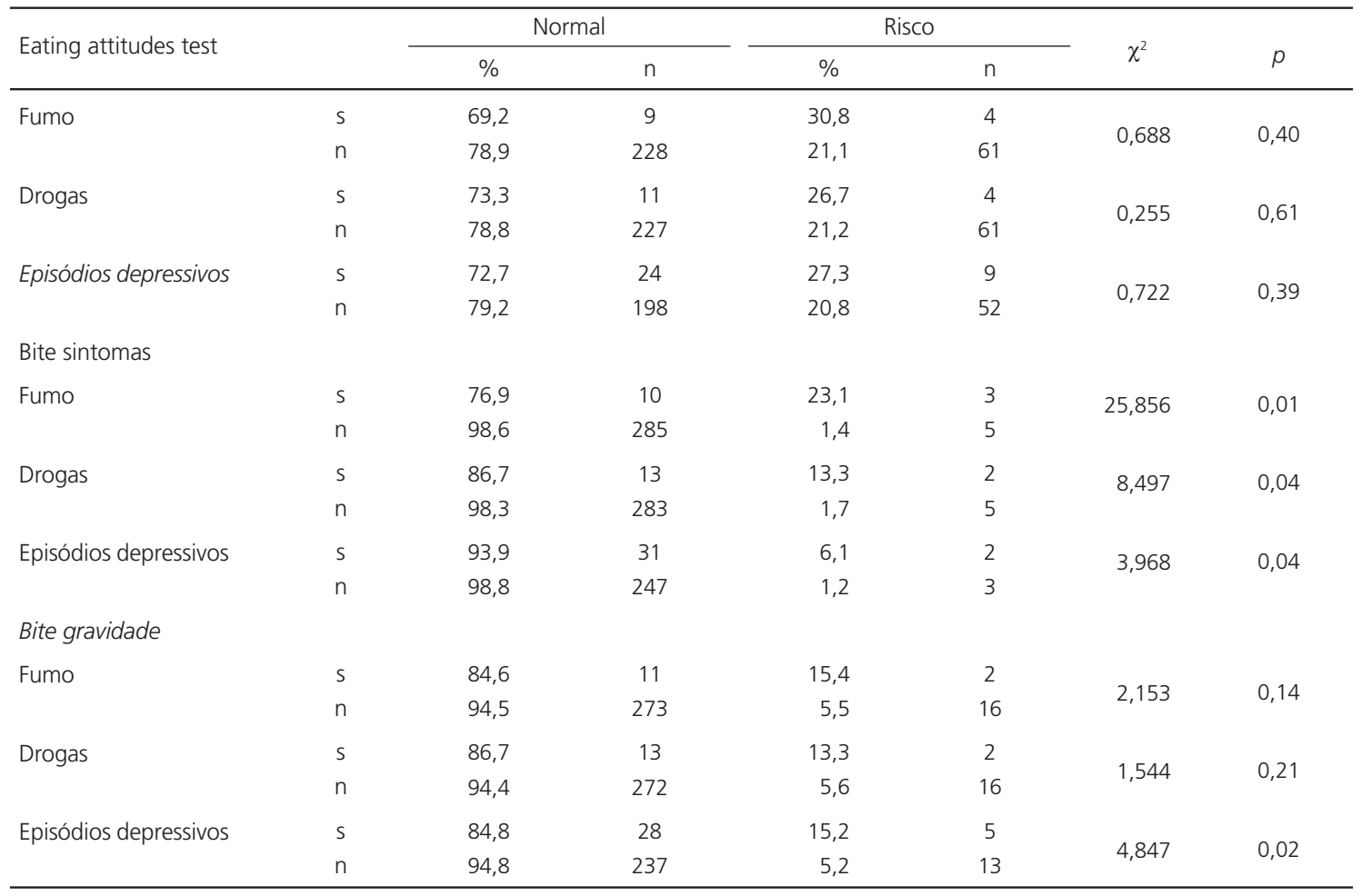


760 | J. P. GOMES et al.

Porém, para o BITE - escala de sintomas, observou-se associação significativa com todas as variáveis analisadas: fumo $\left(\chi^{2}=25,856\right.$ e $\left.p<0,01\right)$, drogas $\left(\chi^{2}=8,497\right.$ e $\left.p<0,04\right)$ e depressão $\left(\chi^{2}=3,968\right.$ e $p<0,04)$. Para o BITE - escala de gravidade, observou-se associação significativa apenas para a variável episódio depressivo $\left(\chi^{2}=4,847\right.$ e $\left.p<0,02\right)$.

\section{I S C U S S Ã O}

A prevalência de alunas com comportamento alimentar compulsivo com grande possibilidade de preencher critérios diagnósticos para bulimia (4,2\%), segundo BITE - escala de sintomas, é semelhante à descrita por Katzman et al. ${ }^{14}$ e Drewnowski et al. ${ }^{15}$. Para o BITE gravidade, apenas 3,6\% das alunas foram classificadas com gravidade significativa/intensa.

Segundo Oliveira ${ }^{16}$, "esses hábitos podem representar o desenvolvimento de síndromes parciais de transtornos do comportamento alimentar, sendo caracterizados como comportamentos alimentares e de risco francamente anormais". O tema tem sido predominantemente tratado em assuntos da adolescência porque se estima que $50 \%$ dos casos de bulimia nervosa ocorram antes dos 18 anos, porém, como o diagnóstico não tem sido fácil nessa faixa etária, tem-se a impressão de sua incidência ser maior acima dessa idade. A média de idade do início da bulimia nervosa foi de 16,3 anos, variando de 13 a 19 anos $^{17}$.

A prevalência de alunas com alto risco de desenvolver anorexia nervosa nesta amostra - 3,6\% - foi menor do que as obtidas por Souza et al. ${ }^{18}$ - 5,5\% - e Vilela et al. ${ }^{3}$ 13,3\% - em estudos com alunas de escolas públicas. Prevalências de sintomas de anorexia nervosa na ordem de $8,8 \%$ ou inferiores são consideradas abaixo da média, indicando uma característica positiva da população estudada em relação aos transtornos alimentares $(18,3)$.

As prevalências de consumo de fumo $(4,2 \%)$ e de drogas $(4,9 \%)$ nesta amostra foram inferiores às prevalências de tabagismo $(12,1 \%)$ encontradas em adolescentes por Malcon, et al. ${ }^{19}$. Um estudo feito por Tavares et al. ${ }^{20}$ mostrou que as substâncias mais consumidas foram álcool $(86,8 \%)$, tabaco $(41,0 \%)$, maconha $(13,9 \%)$, solventes (11,6\%), ansiolíticos (8,0\%), anfetamínicos (4,3\%) e cocaína (3,2\%). Estudos realizados entre escolares de primeiro e segundo graus e entre estudantes universitários mostram, consistentemente, nas diversas regiões do País, que o álcool é a droga mais utilizada, seguido pelo tabaco ${ }^{20}$.

Os solventes se mantêm como os mais comuns no terceiro mundo, após álcool e tabaco, enquanto nos países desenvolvidos a maconha ocupa o terceiro lugar. Deve-se ressaltar que os dados do presente estudo referem-se à amostra estudada de adolescentes escolares e não podem ser extrapolados para a população geral de adolescentes. Contudo, tendo em vista que quase toda a população passa pela escola em idade e circunstância bastante favoráveis à assimilação de novos hábitos e conhecimentos, essa instituição torna-se um espaço privilegiado para o desenvolvimento de programas preventivos, sendo recomendável o estabelecimento de políticas nesse sentido 20 .

O aparecimento de episódios depressivos foi relatado por 10,7\% da amostra. Em pacientes portadores de bulimia nervosa, a incidência de depressão pode variar de 38 a 63\%. Quando se considera a presença de transtornos depressivos em geral, a incidência pode chegar a 83\%, dependendo do estudo ${ }^{21}$. A frequência elevada desses transtornos em pacientes bulímicos levou os pesquisadores a investigarem a relação entre os dois transtornos. Apoiando a relação entre bulimia e depressão, estudos mostraram altas taxas de depressão em parentes de primeiro grau de pacientes bulímicas. Assim como na anorexia, a diminuição do apetite, os hábitos alimentares caóticos e as alterações metabólicas (por exemplo, a depleção de triptofano) são os candidatos mais prováveis para justificar a frequente associação entre bulimia nervosa e depressão ${ }^{21}$.

Nesse sentido, os resultados desse estudo corroboram as evidências da literatura, pois o 
hábito de fumar e consumir drogas apresentou associação significativa com distúrbios alimentares sugestivos de bulimia, tanto para a escala de sintomas $(p<0,04)$ quanto para a escala de gravidade $(p<0,02)$. Porém, os resultados relacionados ao BITE - escala de sintomas apresentaram associação significativa com o consumo de fumo $(0,01)$ e drogas $(0,04)$,

\section{O N CLUS Ã O}

De modo geral, as prevalências de prováveis comportamentos alimentares compulsivos (bulimia e anorexia) estão de acordo com os relatos da literatura. Em relação ao consumo de fumo e drogas, pode-se considerar que as prevalências foram baixas, no entanto, em relação à proporção de jovens que relataram episódios depressivos, a prevalência pode ser considerada elevada, uma vez que foram observadas associações significativas entre episódios depressivos e o BITE, para a escala de sintomas e a escala de gravidade.

Dessa forma, pode-se concluir que a amostra de adolescentes investigada no município de Toledo desenvolveu comportamento de risco relacionado à bulimia nervosa e apresenta índices de sintomas do transtorno, como aqueles observados em outras regiões brasileiras. Os resultados alertam para a necessidade de investimentos em programas de educação nutricional no ambiente escolar com o objetivo de promover mudanças nos conceitos de imagem corporal, conscientizar sobre os prejuízos para a saúde dos comportamentos para redução de peso, além de orientar para escolhas alimentares mais saudáveis.

Esses programas precisam ser dinâmicos, com uma abordagem diferenciada para cada grupo e devem inserir tanto os adolescentes como as crianças em idade escolar, além dos pais, professores e demais profissionais envolvidos com o ambiente escolar.

\section{COLABORADORES}

J. PIAZZON-GOMES e E. LEGNANI contribuiram para o planejamento, concepção do projeto de pes- quisa e do artigo, análise e interpretação dos dados, redação e revisão crítica. R.F.S. LEGNANI contribuiu para o planejamento do artigo, na análise dos dados bem como na organização metodológica do mesmo. N.P. GREGÓRIO contribuiu para o planejamento do artigo, na aplicação dos questionários. R.K. SOUZA contribuiu para o planejamento do artigo, no levantamento bibliográfico e revisão da literatura.

\section{REFERÊ NCIAS}

1. Branco LM, Hilário MOE, Cintra IP. Percepção e satisfação corporal em adolescentes e a relação com seu estado nutricional. Rev Psiq Clín. 2006. 33(6): 292-6.

2. Galindo EMC, Carvalho AMP. Tradução, adaptação e avaliação da consistência interna do Eating Behaviours and Body Image Test para uso com crianças do sexo feminino. Rev Nutr. 2007, 20(1): 47-54. doi: 10.1590/S1415-52732007000100005.

3. Vilela JEM, Lamounier JA, Filho MAD, Barros Neto JR, Horta G.M. Transtornos alimentares em escolares. J Pediatr. 2004, 80(1):49-54.

4. American Psychiatric Association. DSM-IV-TR: manual diagnóstico e estatístico de transtornos mentais. $4^{\mathrm{a}}$ ed. Porto Alegre: Artmed; 2002. p.555-65.

5. World Health Organization. CID-10: classificação de transtornos mentais e de comportamento da CID-10. Descrições clínicas e diretrizes diagnósticas. Porto Alegre: Artmed; 1993 p.173-8.

6. Fiates GMR, Salles RK. Fatores de risco para o desenvolvimento de distúrbios alimentares: um estudo em universitárias. Rev Nutr. 2001; 14(3):3-6. doi: 10.1590/S1415-52732001000400001.

7. Eisler I, Szmukler GI. Social class as a confounding variable in the eating attitudes test. J Psychiatr Res. 1985; 19(2):171-6.

8. Henderson M, Freeman CPL. A self-rate scale for bulimia: the BRITE. Br J Psychiatr. 1987; 150(1): 18-24.

9. Cordás TA, Hochgraf PB. O BITE: instrumento para avaliação de bulimia nervosa - versão para o português. J Bras Psiq. 1993; 42(3):141-4.

10. Gross J, Rosen JC, Leitenberg H, Willmuth ME. Validity of the eating attitudes test and the eating disorders inventory in bulimia nervosa. J Consult Clin Psychol. 1986; 54(1):875-6.

11. Nunes MA, Bagatini LF, Abuchaim $A L$, Kung $A$, Ramos D, Silva JA, et al. Distúrbios da conduta alimentar: considerações sobre o teste de atitudes alimentares (EAT). Rev ABP-APAL. 1994; 16:7-11. 
12. Epidata [acesso 2008 jun]. Disponível em: <http:// www.epidata.dk/>.

13. Organização Mundial de Saúde. Physical status: the use and interpretation of anthropometry. Geneva: WHO; 1995. Technical Report Series n. 854.

14. Katzman M, Wolchik S, Braver T. The Prevalence of frequent binge eating and bulimia in a nonclinical college sample. Int J Eating Disord. 1984; 3(1):53-62.

15. Drewnowski A, Yee DK, Krahn DD. Bulimia in college women. Am J Psychiatry. 1988; 145(6): 7535.

16. Oliveira EA, Santos MA. Perfil psicológico de pacientes com anorexia e bulimia nervosas: a ótica do psicodiagnóstico. Medicina (Ribeirão Preto). 2006; 39(3):353-60.

17. Jones JM, Bennett S, Olmsted MP, Lawson ML, Rodin G. Disordered eating attitudes and behaviours in teenaged girls: a schoolbased study. CMAJ. 2001; 165(5):547-52.
18. Souza FGM, Martins MCR, Monteiro FCC, Menezes Neto GC, Ribeiro IB. Anorexia e bulimia nervosa em alunas da faculdade de Medicina da Universidade Federal do Ceará- UFC. Rev Psiquiatr Clín. 2002; 29(4):172-80, 2002.

19. Malcon MC, Menezes AMB, Chatkin M. Prevalência e fatores de risco para tabagismo em adolescentes. Rev Saúde Pública. 2003; 37(1):1-7.

20. Tavares BF, Beria JU, Lima MS. Prevalência do uso de drogas e desempenho escolar entre adolescentes. Rev Saúde Pública. 2001; 35(2).

21. Alves E, Vasconcelos FAG, Calvo MCM, Neves J. Prevalência de sintomas de anorexia nervosa e insatisfação com a imagem corporal em adolescentes do sexo feminino do Município de Florianópolis, Santa Catarina, Brasil. Cad Saúde Pública. 2008; 24(3).

Recebido em: 17/9/2008

Versão final reapresentada em: 23/7/2009

Aprovado em: 4/5/2010 